\title{
Estimación de consumos de suelo para el servicio público domiciliario de agua ${ }^{1}$
}

\section{SOIL CONSUMPTION FOR THE WATER PUBLIC SERVICES}

ESTIMATIVA DO CONSUMO DE TERRA PARA O SERVIÇO PÚBLICO

DE ABASTECIMENTO DE AGUA

TADEO HUMBERTO Sanabria Artunduaga ${ }^{2}$

Para citar este artículo: Sanabria Artunduaga, T. H. (2018). Estimación de consumos de suelo para el servicio público domiciliario de agua. Perspectiva Geográfica, 23(1), 53-70. doi: 10.19053/01233769.5862

Recibido:

13 de febrero de 2017

Evaluación:

3 de agosto de 2017

Aprobación:

1 de febrero de 2018

\section{Resumen}

El dimensionamiento del consumo de suelo de soporte para el funcionamiento urbano es fundamental para determinar el crecimiento de las ciudades. Este artículo establece un parámetro de medición de ese suelo a partir de la relación entre las demandas de consumo de servicios públicos basados en el agua y los rendimientos hídricos en ocho municipios colombianos. Los resultados establecen un valor de referencia para la estimación del suelo de soporte que vincula la gestión del plan territorial, las áreas protegidas para la producción de los bienes objeto del servicio y la integración con el sistema de movilidad al interior de las ciudades para su distribución a los usuarios.

Palabras clave: consumo de suelo, gestión territorial, servicios públicos domiciliarios, sistema de movilidad, suelo de protección.

$1 \quad$ El artículo se deriva del proyecto de investigación “Aprovechamientos urbanísticos y modelos de ocupación. Estimación de cargas y beneficios urbanísticos a partir de demandas de servicios urbanos".

2 Arquitecto PhD en Gestión y Valoración Urbana de la Universitat Politècnica de Catalunya y Magíster en Ordenamiento Urbano y Regional de la Universidad Nacional de Colombia. Docente investigador de la Universidad de América vinculado al grupo de investigación Prospectiva Urbano Territorial. tadeosan@yahoo.com.mx. 


\section{Abstract}

The dimensioning of the support soil consumption for the urban operation is fundamental to determine the growth cities. This article establishes a parameter of measurement of this land based on the relationship between the consumption demands of domiciliary public services and water yields in eight Colombian municipalities. The results establish a reference value for the estimation of the support land that links to the management of the territorial plan the protected areas to produce the service object and the mobility system integration with of the urban interior for its distribution to users.

Keywords: land use, land management, public utilities, mobility system, soil protection.

\section{Resumo}

O dimensionamento do consumo de piso de apoio para a operação urbana é fundamental para determinar o crescimento das cidades. Este artigo estabelece um parâmetro de medição para este solo, baseado na relação entre as demandas de consumo de serviços públicos baseados em água e a produção de água em oito municípios colombianos. Os resultados estabelecem um valor de referência para a estimativa do solo de apoio que vincula à gestão do plano territorial as áreas protegidas para a produção dos bens objeto do serviço e a integração com o sistema de mobilidade dentro das cidades para sua distribuição para os usuários.

Palavras-chave: consumo de solo, gestão territorial, serviços públicos domésticos, sistema de mobilidade, piso de proteção. 


\section{Introducción}

La planeación territorial ha de responder a varios retos, el primero de los cuales alude a asegurar la atención a las necesidades de los habitantes y con ello a garantizar los servicios que soportan el funcionamiento urbano. Puesto que cada habitante requiere de una cantidad de espacio para desarrollar sus actividades vitales, la concentración permanente de las personas en la ciudad implica que el suelo de los tejidos residenciales es compartido con los suelos dispuestos para la producción y con los suelos que hacen posible su funcionamiento ${ }^{3}$ (Luengo, 2015; Pirez, 2000)

En efecto, la aglomeración urbana implicó el desarrollo de tecnologías y esquemas de administración para asegurar el abastecimiento y la distribución de los servicios vitales, obligando a disponer del suelo para los trazados y las infraestructuras que soportan la habitación en las ciudades. Pero ¿cuánto suelo se ha de disponer para ese propósito?

La literatura revisada a propósito de este documento muestra cierto énfasis en los estudios relativos a los métodos y los modelos de consumo de agua, pero es escaso el abordaje orientado a dimensionar los suelos de protección y los sistemas estructurantes que requiere el ordenamiento territorial para garantizar un soporte suficiente de servicios públicos domiciliarios (SPD). Este artículo propone unos estándares de consumo de suelo para la producción, la distribución y el consumo de SPD basados en el agua a partir de la identificación de patrones de consumo, cuya aplicación permitiría ofrecer una

3 "No existe la vivienda adecuada si no es en conjunción con unos servicios implantados en un el entorno urbano": estos servicios, que incluyen los espacios dedicados para el trabajo, son concebidos como el hábitat urbano (Luengo, 2015, p. 127) referencia de suficiencia funcional al crecimiento de las ciudades en materia de SPD. La hipótesis plantea que las magnitudes de suelo para garantizar una oferta suficiente pueden desbordar las capacidades hídricas de un municipio.

Como el objetivo central de los servicios públicos es el abastecimiento de los bienes y servicios esenciales para la satisfacción de las necesidades individuales, esta atención usualmente alude al abastecimiento de agua y el retorno del agua tratada y consumida (servicios de acueducto y alcantarillado) a sus cuencas, la dotación de energía a los hogares y las instalaciones productivas, la recolección y el tratamiento de basuras y una creciente oferta de nuevos servicios para atender las necesidades de conectividad. La variedad de flujos que movilizan los SPD se traduce en que cada servicio entraña diferentes esquemas de operación y diseño que permiten la producción y la distribución a la población usuaria.

Para dar cuenta del objetivo de este documento, inicialmente se abordan algunas consideraciones conceptuales pertinentes para el cálculo del consumo de servicios, que dan paso a las estimaciones de uso de servicios en los ámbitos normativos y determinan lo que en la literatura urbana se denominan sistemas estructurantes o generales, específicamente los conformados por el suelo y las infraestructuras dedicadas a los SPD, con lo cual se reflexiona sobre el consumo de suelo de los sistemas analizados.

\section{El contexto de prestación de SPD}

Los servicios públicos son mecanismos de producción y distribución de bienes validados culturalmente para satisfacer necesidades que las sociedades han privilegiado como vitales para la reproducción social y económica (Sanabria, 2016), ya que se considera 
que requieren ser consumidos necesariamente y de manera permanente por toda la población y que contribuyen simultáneamente a la satisfacción de varias necesidades.

Su connotación pública deriva de que su producción y su disposición para el consumo requiere del esfuerzo conjunto de la sociedad, para lo cual precisa intervenir tres ámbitos de la realidad: el funcional, el territorial y el normativo. Este último determina las condiciones de acceso de los usuarios al bien satisfactor y las condiciones de distribución del bien para garantizar su disponibilidad y calidad. De ello da cuenta la política pública para la gestión del servicio, que al regular la potencia, la frecuencia y el volumen del suministro a los usuarios determina el dimensionamiento de las infraestructuras de producción, distribución y consumo, y el suelo que será consumido por ellas (Corte Constitucional, 2003; Hurtado González, 2015).

El diseño de estas infraestructuras y sus trazados alude precisamente al ámbito funcional e implica los procesos y los dispositivos técnicos, operacionales, espaciales e instrumentales, de acuerdo con la tecnología disponible, que permiten explotar la fuente del recurso, transformarlo en el bien satisfactor, conducirlo, almacenarlo, distribuirlo a los usuarios, disponerlo para su consumo y utilizarlo. Los servicios públicos toman el carácter de domiciliarios al ser conducidos desde una fuente hasta las residencias, donde son consumidos (Sanabria, 2016).

La localización de las fuentes, las áreas de producción del bien y la de los usuarios está asociada a la propiedad del suelo, que moviliza el libre mercado, por lo que para ejecutar las infraestructuras y los trazados se requiere intervenir el suelo y su propiedad. Las dinámicas de localización de los usuarios del servicio, el suelo consumido por las infraestructuras hincadas sobre él y las relaciones con la renta del suelo y con el derecho de propiedad son operadas en el ámbito territorial, operacionalizado a través del ordenamiento territorial al disponer los suelos requeridos por las infraestructuras, instrumentar la forma de adquirirlos para hacerse a su titularidad y obligar a los promotores inmobiliarios y propietarios del suelo a contribuir con ellos (Olivan, 2007; Solá-Morales, 1997).

El ámbito normativo aporta las indicaciones de consumo individual de los bienes proporcionados bajo un servicio, ofreciendo así las bases de dimensionamiento de la infraestructura; el ámbito funcional aporta los diseños de infraestructura para dar cumplimiento a los estándares de calidad y cantidad del bien suministrado, y el ámbito territorial se refiere a las disposiciones e instrumentos que permiten contar con el suelo para los dispositivos de producción, distribución y consumo, y la ejecución de las obras que materializan el suministro.

\section{Métodos}

La investigación plantea la estimación de los consumos de servicios por habitante dimensionada a partir de estándares que consultan el crecimiento de la población, de lo cual se derivan unos indicadores de consumo de suelo con sus respectivos valores de referencia para la realidad de las ciudades colombianas. Por tratarse de una referencia, su aplicación requiere afinar estos valores desde lo local, pues ha de contemplar especificidades y objetivos propios de ordenación territorial, así como del modelo de ciudad que se desea.

Los análisis de consumo de suelo se derivan de consideraciones teóricas asociadas a las regulaciones urbanísticas y mediciones empíricas que toman ocho casos de estudio, los cuales abarcan desde 
la escala metropolitana a los pequeños poblados: Bogotá, Yopal, San José del Guaviare, El Retorno, Calamar, Marinilla, Itagüí y Chía.

Las variables analizadas resultan de un abordaje conceptual que permite identificar, a partir de los consumos de agua, las infraestructuras que soportan el territorio en lo que respecta a los servicios públicos. El suelo consumido por estas infraestructuras es la base del cálculo, que se discrimina en suelo rural y urbano; los criterios para la propuesta de cálculo son provistos con la explicación del ámbito territorial, donde se concluye que las variables deben permitir una medición del suelo consumido por la operación de cada sistema de servicios públicos y ha de asociarse tanto a la riqueza acuífera de cada localidad como a las demandas ciudadanas.

Los servicios considerados son el de acueducto, el alcantarillado y la energía eléctrica generada hidráulicamente. El consumo de suelo se valora en tres fases de su gestión: la explotación del recurso, la producción del bien y la distribución a los hogares. Para el primer caso, se consideran de manera específica los consumos de agua potable para consumo doméstico y de aguas servidas, orientados a establecer el suelo de protección bajo una relación entre rendimientos hídricos y densidades poblacionales para las zonas de estudio.

Tomando en cuenta los contextos de producción y distribución de los servicios, considerados a partir de datos provistos por diferentes estudios y agencias encargadas de la prestación de servicios públicos, se estableció la extracción necesaria de agua para garantizar el consumo per cápita sugerido por la Comisión Reguladora de Agua Potable y Saneamiento Básico (CRA), el cual se opera con las densidades resultantes de cada localidad estudiada que resultaron de los datos de población reportados por las proyecciones del Departamento Administrativo Nacional de Estadística (DANE) para el año 2015 y de la extensión superficiaria del municipio según sus planes de ordenamiento territorial. Esto define la demanda local de agua potable.

La capacidad hidráulica, por su parte, incorpora los rendimientos hídricos locales al proporcionar una relación entre agua y suelo, pues expresa la oferta de agua por unidad de área de la cual se usan las cantidades que son demandadas. Los datos que reflejan esta condición fueron tomados del Estudio Nacional del Agua (Instituto de Hidrología, Meteorología y Estudios Ambientales [IDEAM], 2015).

Esa demanda corresponde a los consumos de unas poblaciones concentradas bajo ciertas densidades, donde la demanda y la oferta son relacionadas para calcular el suelo rural necesario que asegure dichos consumos. Por lo tanto, estos dos parámetros son operados para estimar el suelo que se requeriría conservar y, por lo tanto, declarar en alguna categoría de protección.

El área que resulta del cálculo corresponde entonces al suelo que se requiere proteger para garantizar los consumos per cápita de agua potable, que se complementa con las franjas hidráulicas que hacen parte del sistema de vertimientos y otras zonas dispuestas para la operación de otros servicios públicos.

También se ofrece una referencia de consumo de los suelos que articulan la distribución de servicios públicos a los sistemas de movilidad en áreas rurales y urbanas a partir de mediciones de los corredores viales en sistemas de información geográficos, al igual que las rondas hidráulicas, establecidas por áreas de influencia de 30 y $50 \mathrm{~m}$, dependiendo de los caudales de la red hidráulica con fuente Open Street Map. Al sumar los resultados del cálculo de 
suelos de protección y de los sistemas de movilidad, tenemos un estimativo del suelo per cápita consumido por los sistemas de servicios públicos cuya operación está basada en el agua.

\section{Los consumos de los servicios asociados al agua en el ámbito normativo}

Algunos SPD como el agua potable, con el respaldo de la Corte Constitucional en 1995, han sido consagrados como derecho fundamental, para cuyo ejercicio el Estado reconoce un mínimo vital bajo el principio de progresividad (Motta, 2011). Pero, por otra parte, es un servicio que atiende las leyes de la oferta y la demanda. De lo anterior se deriva que el servicio pueda ser prestado por agentes privados o por el Estado y que para cuantificar su consumo se acuda a los registros históricos o a los estándares vitales indistintamente.

Si bien la literatura acerca de los consumos per cápita de agua es extensa, también existen parámetros diversos para los consumos básicos que soportan los diseños de las redes. Con respecto al agua potable para supervivencia, la Organización Mundial de la
Salud (OMS, 2009) asume 30 litros mínimos diarios por persona, valor que aumenta a 70 litros diarios al incluir las necesidades de saneamiento.

Pero el agua se requiere también para otras actividades cuyo consumo depende de múltiples factores, entre los que se destaca la actividad económica. Por ejemplo, en oficinas se plantea un consumo estandarizado de 20 litros/día por cada metro cuadrado, lo que equivale a 80 a 100 litros diarios por trabajador, ya que cada trabajador utiliza un área de entre cuatro y cinco $\mathrm{m}^{2}$. Sin embargo, algunas industrias son intensivas en cuanto al consumo de agua y pueden demandar desde 5.000 litros por $\mathrm{m}^{2}$ en un día hasta cerca de $70.0001 / \mathrm{m}^{2}$, como la industria del papel (González, Saldarriaga y Jaramillo, 2010), aunque no toda requiera ser potabilizada.

En estimaciones realizadas por el IDEAM (2015), la demanda de agua en Colombia se representa principalmente mediante los siguientes usos: agrícola, con el $54 \%$ del agua consumida, doméstico, con el $29 \%$, industrial, con el $13 \%$, y pecuario y servicios, con porcentajes del 3 y el $1 \%$ respectivamente. Los consumos por regiones hidrográficas y sectores económicos para el año 2012 se muestran en la Figura 1.

\section{FIGURA 1.}

Distribución porcentual de usos de agua por sectores económicos en cada área geográfica

Fuente: IDEAM (2015, p. 183).

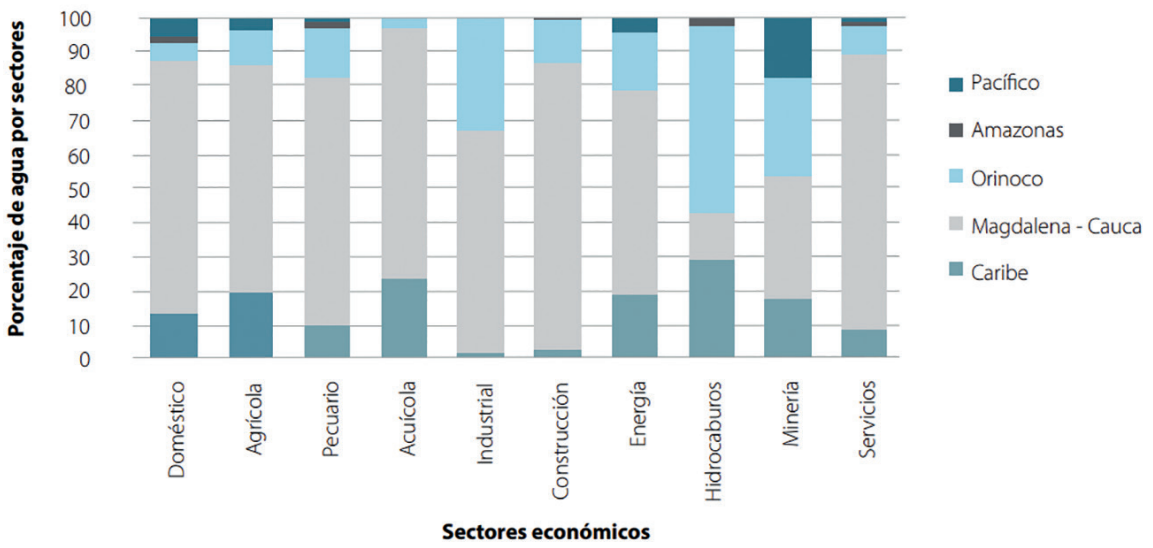


El agua potable presenta la característica de ser un bien inelástico; en consecuencia, las variaciones de consumo por estrato socioeconómico son marginales (Junca, 2000). No obstante, la Figura 2 muestra un incremento notable en los consumos de todas las ciudades para el estrato socioeconómico 6 y un ascenso

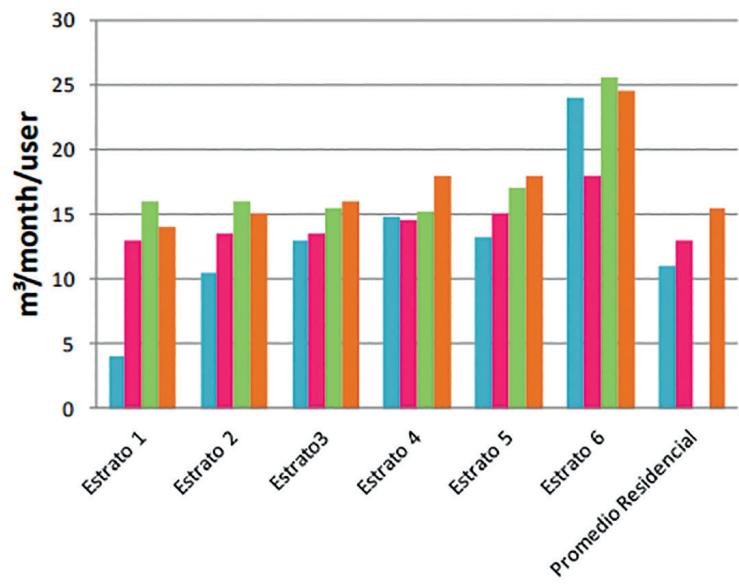

Sin embargo, es más común encontrar referencias del consumo histórico en los hogares, ya que los operadores de los sistemas de acueducto pueden hacer cálculos de consumo a partir de los contadores instalados en en el consumo a medida que se incrementa el estrato en Bogotá. Este resultado puede evidenciar la relación de los consumos con el ingreso, la cual se explica, de acuerdo con la Comisión de Regulación de Agua Potable y Saneamiento Básico (CRA, 1997), por el efecto de la política de subsidios sobre el consumo.

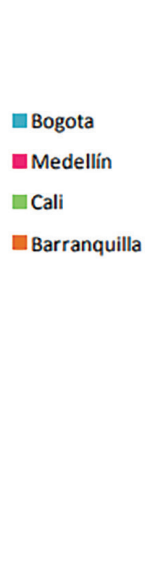

FIGURA 2.

Consumo de agua por unidad.

Fuente: Aguirre (2012, p. 4).

los hogares. Según un estudio del Instituto Nacional de Estadística (INE) de España, los consumos para algunas de las ciudades europeas en comparación con la ciudad de Bogotá, Colombia, son los siguientes:

\begin{tabular}{|c|c|c|}
\hline \multirow{2}{*}{ CIUDAD } & \multirow{2}{*}{$\begin{array}{l}\text { CONSUMO MEDIO } \\
\text { LITROS POR HABITANTE/DÍA }\end{array}$} & \multirow{2}{*}{$\begin{array}{l}\text { CONSUMO MEDIO } \\
\text { M }^{3} \text { POR HOGAR/MES }\end{array}$} \\
\hline & & \\
\hline Oslo & 197 & 23,64 \\
\hline Estocolmo & 178 & 21,36 \\
\hline Lisboa & 159 & 19,08 \\
\hline Londres & 158 & 18,96 \\
\hline Madrid & 131 & 15,72 \\
\hline Bogotá & 146 & 17,48 \\
\hline
\end{tabular}

Fuente: Chacón, Lizcano y Aspilla (2011).

En Colombia, la Empresa de Servicios Públicos de Medellín (EPM, 2009) plantea que una persona consume en promedio $3,8 \mathrm{~m}^{3}$ de agua al mes, es decir que en una familia de cuatro personas (promedio nacional por suscriptor) el consumo promedio mensual debe ser aproximadamente de $15,4 \mathrm{~m}^{3}$. Sin embargo, otros estudios que incorporan incrementos en el consumo por la temperatura arrojan $20 \mathrm{~m}^{3}$ al mes, parámetro de consumo reglado para Colombia por la Comisión Reguladora de Agua Potable y Saneamiento Básico (CRA, 2011), lo que significa 5 $\mathrm{m}^{3} / \mathrm{mes}$ por persona, como se muestra en la Tabla 2 . 
TABLA 2. CONSUMOS DE AGUA SEGÚN FUENTES CONSULTADAS

\begin{tabular}{|c|c|c|c|c|}
\hline DESCRIPCIÓN & EPM & $\begin{array}{c}\text { ÁNGEL, ROZO, } \\
\text { HERNÁNDEZ Y } \\
\text { VALDERRAMA (2002) }\end{array}$ & $\begin{array}{l}\text { DEPARTAMENTO NACIONAL } \\
\text { DE PLANEACIÓN (DNP) }\end{array}$ & $\begin{array}{c}\text { COMISIÓN REGULADORA } \\
\text { DE AGUA POTABLE Y } \\
\text { SANEAMIENTO BÁSICO (CRA) }\end{array}$ \\
\hline $\begin{array}{l}\text { Consumo de } \\
\text { agua/persona }\end{array}$ & $3,8 \mathrm{~m}^{3} / \mathrm{mes}$ & $4,81 \mathrm{~m}^{3} / \mathrm{mes}$ & $5,0 \mathrm{~m}^{3} / \mathrm{mes}$ & $5,0 \mathrm{~m}^{3} / \mathrm{mes}$ \\
\hline \multirow{2}{*}{$\begin{array}{l}\text { Consumo de agua } \\
\text { por suscriptor }\end{array}$} & $15,4 \mathrm{~m}^{3} / \mathrm{mes}$ & $19,25 \mathrm{~m}^{3} / \mathrm{mes}$ & $20,0 \mathrm{~m}^{3} / \mathrm{mes}$ & $20,0 \mathrm{~m}^{3} / \mathrm{mes}$ \\
\hline & & $\begin{array}{l}\text { Con un incremento según } \\
\text { la temperatura del } 20- \\
25 \% \text { en clima cálido }\end{array}$ & $\begin{array}{l}20 \mathrm{~m}^{3} / \mathrm{mes} \\
\text { Promedio entre } 17,7 \text { y } 24,9 \\
\mathrm{~m}^{3} / \text { mes de acuerdo al clima }\end{array}$ & $20,0 \mathrm{~m}^{3} / \mathrm{mes}$ \\
\hline
\end{tabular}

Fuente: elaboración propia.

El ciclo del consumo de agua de uso doméstico, industrial, comercial e institucional concluye con el retorno a las cuencas, lo cual obliga a incluir la cuantificación de los caudales de diseño del acueducto en los sistemas de alcantarillado. Las aguas residuales son conducidas desde donde se originan hasta donde son tratadas y depositadas en las fuentes, pero como las aguas no consumidas incrementan los volúmenes conducidos por las redes podrían ser tratadas diferencialmente, pues su separación excluye el agua de escorrentía del tratamiento al que es sometida el agua residual doméstica.

Lo anterior significa que si bien la separación de las aguas residuales y aguas lluvias en los sistemas de alcantarillado es deseable, los reglamentos de este servicio permiten redes combinadas, entre otras razones, porque aun operan en las zonas urbanas, especialmente en las antiguas. Cualquier sistema, sin embargo, requiere ser calculado a partir de los caudales máximos esperados (Ministerio de Vivienda, Ciudad y Territorio, 2012).

El agua participa también en la generación de energía eléctrica con un $70,41 \%$ del total, según datos del sistema de información de la Unidad de Planeación Minero-Energética (UPME, 2013). La energía restante es generada por combustibles de- rivados del petróleo en un 27,23 \% y, en una ínfima pero creciente fracción, un 2,37\% por sistemas de energías alternativas limpias, como las solares, las eólicas o las geotérmicas y biomasa.

Según datos del Banco Mundial, la demanda de energía es creciente: el consumo mundial per cápita para el 2013 fue de $3.104 \mathrm{kWh}$ y de $1.177 \mathrm{kWh}$ en Colombia; de este, el consumo residencial representa algo más del $42 \%$ y se concentra especialmente en grandes ciudades y en estratos medios (Aguirre, 2012). Según la EPM (2009), el consumo mensual residencial para una familia de cuatro personas en promedio debe ser $152 \mathrm{kWh} / \mathrm{mes}$, lo cual representa cerca de $40 \mathrm{kWh}$ por persona. Pero producir un $\mathrm{kWh}$ con tecnología hidráulica depende del caudal y de la altura de caída: por ejemplo, una caída de $20 \mathrm{~m}$ y un caudal de 16 litros/segundo genera 1,5 kWh.

\section{El ámbito funcional de los servicios públicos relacionados con el agua}

Todas las guías para el diseño de acueductos señalan como primer paso la definición de la complejidad del sistema, la cual es determinada por el tamaño de la 
demanda, el nivel socioeconómico de los usuarios y la dificultad técnica que entraña su ejecución y su operación. En un sistema altamente complejo hay un incremento hasta del $50 \%$ en la dotación por habitante si se compara con un sistema de complejidad bajo (Lee y Jouravlev, 1998). El cálculo de la demanda, de acuerdo con el Reglamento Técnico del Sector de Agua Potable y Saneamiento Básico (RAS), tiene en cuenta lo que la población consume ${ }^{4}$, pero también la oferta del agua, esto es, las fuentes de abastecimiento.

Sin embargo, debido a las pérdidas en la operación del tratamiento del agua y las comerciales y técni- cas en la red de conducción, no toda el agua que se consume es la que sale de la fuente. El ChartsBin Statistics Collector Team (2011) y Ramírez (2014), entre otros, estimaron que el $45 \%$ del agua producida en América Latina es agua no facturada, y se afirma que de 470 litros/persona/día extraídos de la fuente solo 309 son tratados. De estos se consumen 247 litros, de los cuales hay 150 que corresponden al consumo residencial (DNP y Banco Mundial, 2014; Sánchez, Flórez y Mejía, 2002). Así, los sistemas de acueducto captan casi tres veces lo que realmente consumen los usuarios, como se ilustra en la Figura 3.

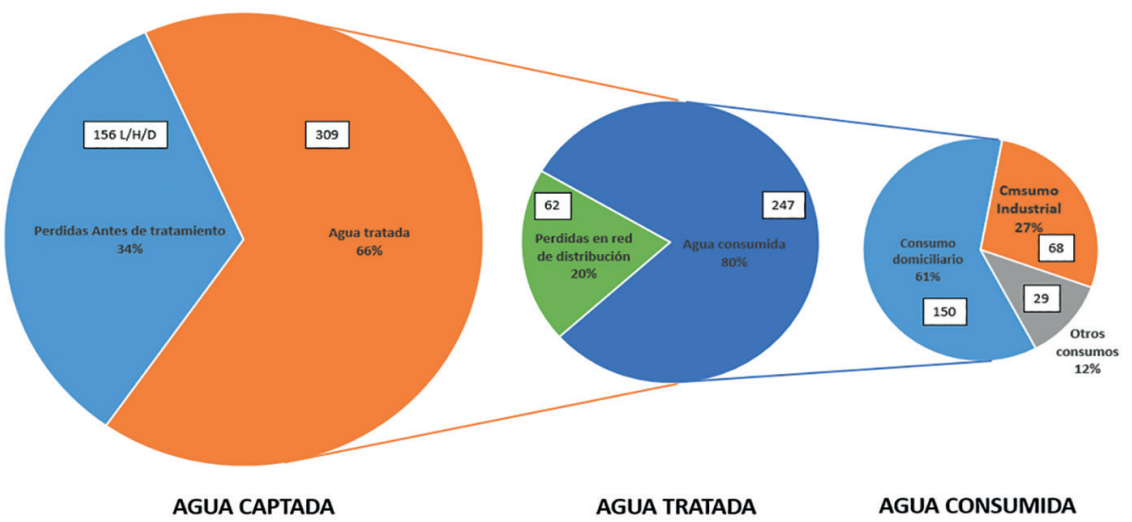

\section{FIGURA 3.}

Volúmenes de agua doméstica desde el abastecimiento al consumo (litros/hombre/día).

Fuente: elaboración propia con base en datos del Departamento Nacional de Planeación (DNP) y el Banco Mundial (BM) (2014).
Con lo anterior se definen los parámetros de diseño que se expresan en las infraestructuras que soportan la oferta del servicio de acueducto. Este sistema requiere dispositivos que permitan la extracción (bocatomas), la potabilización (plantas de tratamiento de agua potable), el almacenamiento (tanques de almacenamiento) y la distribución primaria en suelo rural, que se conecta a las redes secundarias y domiciliarias en suelo urbano. Generalmente,

4 Los consumos proyectados toman en cuenta las densidades de población. Se calcula la densidad de acuerdo a los estándares de dotación por suscriptor, que coincide con cada acometida domiciliaria; por tanto, cada usuario del sistema corresponde a un hogar y uso. estas últimas vinculan el esfuerzo público y privado necesario para el consumo de agua potable a través de las acometidas a cada una de las residencias.

La red de alcantarillado sanitario, por su parte, está compuesta por una red de tuberías que se instalan a determinada profundidad en las vías públicas, conectando las acometidas domiciliarias de aguas servidas con colectores de diferente nivel hasta el punto donde son tratadas y vertidas nuevamente a la red hidrológica para ser reutilizadas.

El sistema de energía eléctrica generada bajo cualquier tecnología en Colombia está interconectado en 
red, lo cual supone un mismo sistema de distribución que vincula las plantas de generación térmica o hidráulica con los usuarios finales (UPME, 2013). La infraestructura de conducción que soporta elevados flujos de energía en áreas rurales es aérea y está soportada por torres. Cuando ingresa a los asentamientos, la tensión se reduce significativamente y se mantienen unas redes aéreas en corredores de media tensión, ligadas a subestaciones que distribuyen la energía a redes de baja tensión. Estas redes son dispuestas sobre el tramado vial, desde donde se les liga a las acometidas de cada residencia (World Bank Group, 2002). Las redes que configuran el sistema de movilidad en la ciudad tienen, además de su función natural de conexión, la de transportar los flujos de los bienes que son el objeto del consumo de los SDP y distribuirlos entre sus usuarios.

\section{El consumo de suelo y el ámbito territorial}

La integración de los SPD al sistema de movilidad implica que este último determine principalmente el consumo de suelo en las ciudades. El sistema de drenaje del agua de escorrentía, aunque puede hacer parte del sistema de movilidad, se incorpora a la red de caños y quebradas urbanas que constituyen la estructura ecológica principal, y el consumo de suelo relativo al alcantarillado pluvial incluye a las áreas protegidas urbanas.

Como el agua es un recurso natural que requiere ser abastecido, la primera demanda de suelo está asociada con su producción y su transformación en los bienes que serán ofrecidos, la segunda, con su tratamiento para garantizar la calidad, y una última, con las infraestructuras para su distribución a los usuarios.
Para dimensionar los consumos de suelo, una aproximación a la primera demanda está vinculada a la huella ecológica, un sistema de medición que indica los recursos naturales utilizados para sostener el actual estilo de vida. Su práctica se ha orientado a medir el hinterland relativo a la producción de bienes en función del recurso utilizado, por lo cual los estudios de la huella ecológica derivaron hacia la huella hídrica y la huella de agua. La primera, de acuerdo a la definición de Hoekstra, Chapagain, Aldaya y Mekonnen (2011), es la cantidad de agua necesaria para producir un producto y asimilar los contaminantes que se generan a partir de él. La segunda incorpora los impactos que resultan en el ciclo de vida de los productos, específicamente, la huella hídrica azul, que está asociada con la extracción de agua superficial y subterránea ${ }^{5}$. Infortunadamente, estas medidas no permiten establecer estimación alguna de la superficie de suelo consumida, sino del agua necesaria para sostener nuestro estilo de vida.

Sin embargo, se cuenta con una política de aseguramiento de las áreas de abastecimiento hídrico que se corresponde con una declaratoria como área de protección, la cual implica la restricción de actividades privadas y su gestión pública e indica que dicha área debe ser enajenada. Esto significa que es preciso identificar y delimitar esas áreas cuyo tamaño está en relación con los objetivos de la conservación, que son múltiples y variados.

Entre estos objetivos, los decretos 462 de 2008 y 2372 de 2010 les asigna los de preservación o res-

5 La huella hídrica azul se refiere a la parte que no retorna a la cuenca de la que se extrajo o retorna con una calidad diferente a la original. La huella hídrica de Colombia es de $812 \mathrm{~m}^{3}$ al año por persona, mientras que el promedio mundial es de $1.243 \mathrm{~m}^{3}$ al año por persona. Si se considera que un valor alto de huella hídrica en relación con la oferta disponible es un indicador de vulnerabilidad del recurso hídrico en una cuenca, se podría decir que la huella hídrica de los colombianos aún no es alarmante. 
tauración de las condiciones naturales, del paisaje o de la capacidad productiva de los ecosistemas para regular la oferta de bienes y servicios ambientales y la preservación de la biodiversidad, de sus manifestaciones singulares y de los espacios naturales asociados a expresiones étnicas y culturales, como complemento a la prevención de riesgos naturales. A pesar de que todos ellos tienen relación con el agua $^{6}$, algunas figuras como la reserva forestal protectora y el distrito de manejo integrado están más estrechamente vinculados a objetivos de regulación hídrica, producción y consumo de agua (Ministerio de Ambiente, Vivienda y Desarrollo Territorial [MAVDT], 2010).

El área protegida y las medidas de protección se adoptan para salvaguardar que dicha fuente asegure como mínimo los caudales de diseño, condición dependiente de la abundancia hídrica que expresa la densidad de la red fluvial superficial y de cuerpos laminares (Sistema de Información Ambiental de Colombia [SIAC], 2002). Pero como la proporcionalidad entre los consumos de agua y de suelo de protección de las fuentes está determinada por las solicitudes de captación y estas, a su vez, por los caudales, el tamaño y distribución de las áreas de protección es diversa.

La estimación de la abundancia o escasez es resultado de medidas de escorrentía y de rendimiento que proporcionan una relación entre agua y suelo, pero resulta ser tan heterogénea como la distribución de las actividades económicas y de la población. El Estudio Nacional del Agua (ENA) 2014, publicado por el IDEAM (2015), señala un rendimiento

6 La gestión de las autoridades ambientales, de acuerdo con la Política Nacional para la Gestión Integral del Recurso Hídrico (MAVDT, 2010), ha estado centrada en el agua que ha sido reconocida como el eje de la gestión ambiental. hídrico estimado entre los 116 hasta los 151/s/ $\mathrm{km}^{2}$ en el centro andino y muestra que los municipios con alto consumo poseen menos cantidad de agua de la demandada.

Si esa demanda corresponde a la huella hídrica, se podrán determinar insuficiencias en el abastecimiento. El IDEAM estima una disponibilidad de $2.830 \mathrm{~m}^{3}$ por persona al mes, que se reduce a 2.225 $\mathrm{m}^{3}$ en un año seco. El resultado del análisis a nivel nacional realizado por el ENA (IDEAM, 2015) permite estimar una disponibilidad de agua azul aproximadamente 100 veces superior a la demanda (huella hídrica azul). No obstante, en varias zonas se evidencian conflictos por el uso y concentración de los consumos.

\section{Resultados}

En el apartado anterior se ha expresado que las medidas de protección se concentran en mantener la oferta de agua y que para ello se utilizan instrumentos tales como la declaración de áreas de protección de las zonas de recarga hídrica y de las rondas de los cuerpos de agua ${ }^{7}$. Estas áreas tendrán correspondencia tanto con los rendimientos hídricos locales como con su huella hídrica.

Si se considera una medida de consumo per cápita domiciliaria de 166 litros/día por persona, lo cual supone una extracción de $8,55 \mathrm{~m}^{3} /$ habitante, y la traducimos al área superficial que se requiere para mantener dicho consumo de acuerdo a los rendimientos hídricos de cada unidad de estudio, se podrá estimar la magnitud de superficie de suelo requerida para conservar la oferta de agua potable de esa zona.

7 Regulada nacionalmente en $15 \mathrm{~m}$ de distancia mínima a cada lado de sus cotas de inundación. 
TABLA 3. CALCULO DEL ÁREA MÍNIMA DE CONSERVACIÓN REQUERIDA PARA GARANTIZAR LOS CONSUMOS RESIDENCIALES DE AGUA POTABLE

\begin{tabular}{|c|c|c|c|c|c|c|}
\hline Municipio & $\begin{array}{l}\text { Rendimiento } \\
\text { hídrico en un año } \\
\text { medio por km }\end{array}$ & $\begin{array}{l}\text { Densidad de } \\
\text { población }(*)\end{array}$ & $\begin{array}{l}\text { Extracción } \\
\text { total } \\
\text { requerida }\end{array}$ & $\begin{array}{l}\text { Consumo/ } \\
\text { oferta }\end{array}$ & $\begin{array}{c}\text { Área } \\
\text { municipal }(*)\end{array}$ & $\begin{array}{c}\text { Área a } \\
\text { conservar }\end{array}$ \\
\hline & $\mathrm{M}^{3} / \mathrm{mes}$ & $\mathrm{Hab} . / \mathrm{km}^{2}$ & $\mathrm{M}^{3} /$ mes & $\mathrm{M}^{3} / \mathrm{mes}$ & $\mathrm{Km}^{2}$ & $\mathrm{Km}^{2}$ \\
\hline Marmato (Caldas) & 116.640 & 237 & 2.025 & 57,59 & 38 & 0,7 \\
\hline Bogotá (municipio) & 57.024 & 4.996 & 42.716 & 1,33 & 1.617 & $1.211,6$ \\
\hline Bogotá (urbano) & 57.024 & 16.335 & 139.667 & 0,41 & 493,7 & 22,00 \\
\hline Chía & 57.024 & 1.636 & 13.987 & 4,08 & 81 & 19,9 \\
\hline Itagüí & 116.640 & 12.989 & 111.058 & 1,05 & 21 & 20,1 \\
\hline Yopal & 158.967 & 59 & 501 & 317,10 & 2.493 & 7,9 \\
\hline Calamar & 187.116 & 1 & 6 & $33.688,31$ & 13.994 & 0,4 \\
\hline El Retorno & 155.520 & 2 & 16 & $9.773,31$ & 12.345 & 1,3 \\
\hline San José del Guaviare & 155.520 & 4 & 34 & $4.610,87$ & 16.632 & 3,6 \\
\hline
\end{tabular}

(*) Los datos consignados corresponden a lo indicado por proyecciones del Departamento Administrativo Nacional de Estadística (DANE) para el año 2015, y la extensión superficiaria del municipio ha sido tomada de sus planes de ordenamiento territorial.

Fuente: elaboración propia.

\section{Análisis de los resultados}

De acuerdo con los resultados, la oferta de agua local en un año medio es suficiente para todos los municipios incluidos en la Tabla 3; sin embargo, se debe señalar que en un año seco la oferta se reduce significativamente. La diferencia entre el municipio y el área urbana de Bogotá, que para el área urbana arroja un déficit superior al $40 \%$, se explica por las grandes diferencias en la superficie y en las densidades, por lo cual en estos casos es necesario completar los requerimientos de agua desde otras jurisdicciones y se hace indispensable una gestión regional del recurso, que implica implementar medidas de protección del suelo para garantizar una oferta suficiente de agua. Para ofrecer este soporte, además de las declaraciones del suelo de protección, se añaden otras figuras que no son estrictamente de protección en el medio nacional, como las áreas para infraestructura de servicios, las cuales reservan suelo para la instalación de infraestructuras de servicios públicos y restringen totalmente cualquier ocupación.

Los suelos bajo estas figuras legales permiten incluir áreas para soportar otros servicios públicos y amortiguar su afectación, entre ellos, la disposición y tratamiento de las 0,27 toneladas anuales de residuos sólidos por habitante o de otros elementos dados de baja en la ciudad, con lo cual las áreas protegidas, sumadas a las reservadas bajo la figura legal citada, constituyen la razón y la base del dimensionamiento del consumo de suelo rural para los SPD. La cantidad de suelo no puede ser inferior al necesario para soportar las demandas per cápita de agua en función del rendimiento hídrico local.

Al comparar los datos de las áreas protegidas declaradas según el Registro Único Nacional de Áreas Protegidas (RUNAP) (Parques Nacionales Naturales de Colombia, 2015) con los requerimientos de suelo de protección para garantizar exclusivamente los consumos de agua potable, se observa que, no obstante la enorme variabilidad en las áreas protegidas municipales, el promedio nacional de las áreas de protección supera ampliamente las necesidades de conservación. Sin embargo, en los municipios con predominio urbano el suelo con declaratoria de protección es insuficiente para garantizar los servicios básicos. 
TABLA 3. COMPARATIVO DE ÁREAS DE PROTECCIÓN DECLARADAS Y NECESIDADES DE PROTECCIÓN DEL SUELO PARA LA ATENCIÓN DE SERVICIOS PÚBLICOS RELACIONADOS CON EL AGUA POTABLE

\begin{tabular}{|c|c|c|c|c|c|c|}
\hline Municipio & $\begin{array}{l}\text { Población } \\
\text { total }(*)\end{array}$ & $\begin{array}{c}\text { Área } \\
\text { Municipal } \\
\left({ }^{*}\right)\end{array}$ & $\begin{array}{c}\text { Área requerida } \\
\text { a conservar } \\
\text { por habitante }\end{array}$ & $\begin{array}{c}\text { Área } \\
\text { Protección } \\
\text { Total }(*) \\
\end{array}$ & $\begin{array}{c}\% \text { suelo } \\
\text { municipal de } \\
\text { protección }\end{array}$ & $\begin{array}{l}\text { Área Protegida } \\
\text { per cápita }\end{array}$ \\
\hline & Hab. & $\mathrm{Km}^{2}$ & $M^{2}$ & $\mathrm{Km}^{2}$ & $\%$ & $M^{2}$ \\
\hline Marmato (Caldas) & 9.096 & 38,40 & 73,30 & 0,04 & 0,11 & 4,53 \\
\hline Bogotá & 8.080 .734 & $1.617,43$ & 149,94 & 729,67 & 45,11 & 107.9 \\
\hline Chía & 132.691 & 81,11 & 149,94 & 19,31 & 23,80 & 145,49 \\
\hline Itagüí & 273.944 & 21,09 & 73,30 & 4,02 & 19,08 & 14,69 \\
\hline Yopal & 146.204 & $2.493,49$ & 53,78 & 107,97 & 4,33 & 738,46 \\
\hline Calamar & 9.091 & $13.994,10$ & 45,69 & $4.179,43$ & 29,87 & $459.733,20$ \\
\hline El Retorno & 22.975 & $12.344,60$ & 54,98 & $6.495,16$ & 52,62 & $282.705,36$ \\
\hline San José del Guaviare & 65.611 & $16.631,80$ & 54,98 & 537,69 & 3,23 & $8.195,09$ \\
\hline Promedio & $1.092 .543,25$ & $5.902,75$ & 81,99 & $1.509,16$ & 22,27 & $93.953,39$ \\
\hline
\end{tabular}

(*) Los datos consignados corresponden a lo indicado por proyecciones del Departamento Administrativo Nacional de Estadística (DANE) para el año 2015 y la extensión superficiaria del municipio, así como su área de protección ha sido tomada de sus planes de ordenamiento territorial.

Fuente: elaboración propia.

En Bogotá, según datos de la Secretaría de Planeación Distrital, el 13,5\% del suelo urbano es de protección, mientras que en el caso del suelo rural corresponde al $59 \%$. El alto porcentaje de suelo rural protegido obedece a la presencia del páramo de Sumapaz y las áreas de reserva de los cerros tutelares. Lo anterior significa que a cada bogotano le corresponden actualmente $7,70 \mathrm{~m}^{2}$ de suelo urbano y cerca de $100 \mathrm{~m}^{2}$ de suelo rural, pero el área que se debería proteger en realidad correspondería a $150 \mathrm{~m}^{2}$.

Si se comparan las cifras de las áreas protegidas declaradas $^{8}$ con los $81,99 \mathrm{~m}^{2}$ del promedio que resulta del cálculo del área de protección requerida por habitante para los ocho municipios que se estudiaron (Tabla 3), se puede afirmar que el área protegida localmente en suelo rural no puede ser inferior a la que permite garantizar $\operatorname{los} 5 \mathrm{~m}^{3}$ de agua

8 La fuente de datos principal fue el aplicativo del Sistema de Información Ambiental para Colombia (SIAC), con datos del Sistema Nacional de Áreas Protegidas (SINAP), y complementado con documentación de los planes de ordenamiento territorial (POT) locales. potable mensuales a su población en función de los rendimientos hídricos locales. Para los casos donde esta relación está por debajo, como sucede en Bogotá, Chía e Itagüí, se impone la necesidad de incrementar las áreas protección, incluso por fuera de su jurisdicción, y para garantizar el consumo mensual de cada $\mathrm{m}^{3}$ de agua es necesario proteger, en los municipios analizados, $16,3 \mathrm{~m}^{2}$ de suelo por habitante.

De otra parte, la riqueza hidráulica y la densidad son determinantes, pues al comparar las rondas hidráulicas de los municipios y el suelo requerido para la producción de agua potable la variabilidad es enorme, ya que mientras en Itagüí las áreas per cápita de ronda son inferiores a $1 \mathrm{~m}^{2}$, en los municipios del Guaviare (Calamar, Retorno y San José) son superiores a $5.000 \mathrm{~m}^{2}$. Lo anterior se añade a la dificultad para las mediciones de las rondas exclusivamente utilizadas para los sistemas de vertimientos y las áreas para su tratamiento, ya que en los planes de ordenamiento territorial (POT) respectivos estas 
áreas no están discriminadas y esto obligaría a revisar si están incorporadas en el estándar de consumo de áreas protegidas aquí provisto, lo cual escapa a los análisis realizados.

Al irrumpir en la ciudad, todos los SPD citados se integran a las vías o necesitan de la conectividad que estas proporcionan. En Colombia, se puede establecer el porcentaje de suelo destinado a la movilidad al estimar el número de kilómetros de la malla vial vehicular y relacionarlo con la superficie urbana, como planteó Fajardo (2005), que en su estudio sobre Medellín calculó que el $22,18 \%$ del suelo urbano correspondía a su infraestructura vial; con esto podemos deducir que cada ciudadano de Medellín disponía para ese año de $9,58 \mathrm{~m}^{2}$ por habitante. Este valor se aproxima al deducido por Alcántara (2010), quien infiere un rango de consumo de suelo para el sistema de movilidad de entre 11,85 y 13,50 $\mathrm{m}^{2}$ por habitante para densidades habitacionales de 40 a 60 viviendas por hectárea.

Bajo este escenario, el promedio de las superficies requeridas para los servicios analizados, las cuales están integradas por las áreas de protección y el área consumida por el sistema vial para su distribución, se muestra en la Tabla 4.

\section{TABLA 4. REQUERIMIENTOS PER CÁPITA DE ÁREAS DE PROTECCIÓN Y DISTRIBUCIÓN PARA SPD ASOCIADOS AL AGUA}

\begin{tabular}{lrrrr} 
Municipio & Población total & $\begin{array}{c}\text { Protección per cápita: } \\
\text { extracción-producción } \\
\text { M2/hab. }\end{array}$ & $\begin{array}{c}\text { Distribución per cápita: } \\
\text { sistema de movilidad } \\
\text { M2/hab. }\end{array}$ & $\begin{array}{c}\text { Área total consumida } \\
\text { Marmato }\end{array}$ \\
Hab. & 9.096 & 73,3 & 41,82 & 115,12 \\
Bogotá & 8.080 .734 & 149,94 & 16,22 & 166,16 \\
Chía & 132.691 & 149,94 & 43,42 & 193,36 \\
Itagüí & 273.944 & 73,3 & 8,74 & 82,04 \\
Yopal & 146.204 & 53,78 & 126,75 & 180,53 \\
Calamar & 9.091 & 45,69 & 374,29 & 419,98 \\
El Retorno & 22.975 & 54,98 & 312,31 & 367,29 \\
San José del Guaviare & 65.611 & 54,98 & 294,42 & 349,40 \\
Total general & 1.092 .543 & 81,99 & 172,07 & 254,06 \\
\hline
\end{tabular}

Fuente: elaboración propia.

\section{Conclusiones}

Con base en los análisis realizados para los ocho municipios, al sumar el promedio del balance del rendimiento y el consumo de agua con el suelo utilizado para su distribución en la ciudad, que está integrado por el tramado de sus vías, podría entonces sugerirse un valor de referencia per cápita de $95 \mathrm{~m}^{2}$ de suelo dedicados a la prestación de los SPD asociados al agua.
Del total de este suelo, $13 \mathrm{~m}^{2}$ corresponden a la distribución urbana del servicio, a la que se sumarían $7 \mathrm{~m}^{2}$ de suelo de protección de los corredores hidráulicos en su interior, es decir, $20 \mathrm{~m}^{2}$ por habitante. El suelo para garantizar la captación, la transformación y las redes matrices en las áreas rurales corresponde entonces a un mínimo de 75 $\mathrm{m}^{2}$ per cápita que debe ser incluido en alguna de las categorías de protección. 
Se pone de relieve el hecho de que la distribución no homogénea de la riqueza hídrica en Colombia impone una planificación regional del recuso agua, cuestión en la que ha insistido reiteradamente la comunidad académica y que ya tiene desarrollos normativos con los planes de manejo de cuencas hidrográficas, pero que deben articularse a las regulaciones del suelo que, en función de su autonomía, ejercen los municipios a través de sus planes de ordenamiento territorial (POT). Esa interacción posibilita el balance regional de la oferta hídrica y las medidas de protección del suelo para garantizar una oferta constante. Los estándares aquí aportados y el método propuesto para determinarlos podrían contribuir a facilitar el establecimiento de determinantes de protección para los POT.

Sin embargo, estos valores son solo referenciales, pues han de revisarse para cada caso los rendimientos hídricos del municipio frente a los consumos esperados de los usuarios para todos los servicios y no únicamente los relativos al consumo de agua residencial, como se ha hecho en este estudio. Como el ejercicio, en este caso, solo evalúa el suelo consumido por el consumo doméstico de agua, sería necesario agregar el suelo para los consumos de agua requeridos para propósitos productivos, las áreas de los drenajes para los servicios de alcantarillado pluvial (cuerpos de agua urbanos y rurales), sus respectivos corredores de protección del cauce y el suelo para soportar la infraestructura de generación eléctrica y el manejo de residuos sólidos.

El ejercicio, además de reiterar la necesidad de preservar el suelo necesario para la adecuada provisión (presente y futura) de los servicios públicos, sugiere con estos resultados la utilización de mecanismos e instrumentos legales para una gestión regional de los suelos que garantice y proteja el agua para la producción de los bienes que constituyen los servicios, y de instrumentos de gestión local sobre el suelo urbano para garantizar la distribución a los usuarios con la integración de los múltiples servicios al sistema de movilidad. 


\section{Referencias}

Aguirre, M. F. (2012). La construcción sostenible en Colombia, presente y futuro. Revista Informe Económico Camacol, 40, 1-7. Recuperado de https://camacol.co/ internas/la-construcci\%C3\%B3n-sostenible-en-colombia-presente-y-futuro

Alcántara, E. (2010). Consumo de recursos en la movilidad. En Análisis de la movilidad urbana. Espacio, medio ambiente y equidad (pp. 77-99). Bogotá: CAF. Recuperado de https://www.caf.com/media/3155/An\%C3\%A1lisis_movilidad_urbana.pdf

Ángel, J., Rozo, J., Hernández, L. y Valderrama, D. (2002). Estimación del consumo básico de agua potable en Colombia. Revista Regulación de Agua Potable y Saneamiento Básico, 7, 15-42. Recuperado de https://doctrina. vlex.com.co/vid/estimacion-basico-agua-potable-colombia-430525978? ga $=2.190105646 .44418148 .1513100570-2065719251.1506542253$

Chacón, G., Lizcano, I y Aspilla, Y. (2011). Consumo básico de agua potable en Colombia. Tecnogestión,8(1), 14-23. Recuperado de http://revistas.udistrital.edu. co/ojs/index.php/tecges/article/view/4379/6601

ChartsBin Statistics Collector Team. (2011). Total Water Use per capita by Country. Recuperado de http://chartsbin.com/view/1455

Comisión de Regulación de Agua Potable y Saneamiento Básico (CRA). (1997). Análisis del sector de agua potable y saneamiento en Colombia. Plan Regional de Inversiones en Ambiente y Salud. Serie Análisis n. ${ }^{\circ} 11$. Recuperado de http://www. paho.org/col/index.php?option =com_docman\&view = download\&category slug $=$ documentos-nacionales\&alias $=1392$-analisis-sectorial-de-agua- $y$ saneamiento-en-colombia\&ltemid $=688$

Corte Constitucional de la República de Colombia. (2003). Sentencia C-150/03. Artículo $2^{\circ}$. Intervención del Estado en los servicios públicos y libre empresa.

Decreto 462. (23 de diciembre de 2008). Por el cual se adopta la Política para el Manejo del Suelo de Protección en el Distrito Capital. Recuperado de http://www. alcaldiabogota.gov.co/sisjur/normas/Norma1.jpp?i $=34288$

Decreto 2372. ( $1^{\circ} \mathrm{de}$ julio de 2010). Por el cual se reglamenta el Decreto-ley 2811 de 1974, la Ley 99 de 1993, la Ley 165 de 1994 y el Decreto-ley 216 de 2003, en relación con el Sistema Nacional de Áreas Protegidas, las categorías de manejo que lo conforman y se dictan otras disposiciones. Diario Oficial n. ${ }^{\circ} 47757$.

Departamento Nacional de Planeación (DNP) y Banco Mundial (BM). (2014). Estrategia Nacional para el Desarrollo de Infraestructura. [Informe preliminar]. Bogotá, D. C.: DNP y BM.

Empresas Públicas de Medellín (EPM). (2009). Normas de Diseño de Sistemas de Acueducto de EPM. Medellín: Empresas Públicas de Medellín. Recuperado de https://www.epm.com.co/site/Portals/0/centro_de_documentos/ NormasDisenoSistemasAcueducto.pdf

Fajardo, S. (2005). La capacidad de soporte del territorio, un instrumento para definir las condiciones de habitabilidad de la ciudad en el Plan de Ordenamiento 
Territorial de Medellín. Ib Revista de la Información Básica, 2(1). Recuperado de https://sitios.dane.gov.co/revista_ib/html_r2/articulo3_r2.htm

González, M. C., Saldarriaga, G. y Jaramillo O. (2010). Estimación de la demanda de agua. Conceptualización y dimensionamiento de la demanda hídrica sectorial. Estudio Nacional del agua 2010 (pp. 170-228). Bogotá: IDEAM. Recuperado de http://documentacion.ideam.gov.co/openbiblio/bvirtual/021888/021888.htm

Hoekstra, A., Chapagain, A. K., Aldaya M. M. y Mekonnen, M. M. (2009). The Water Footprint Assessment Manual. Setting the global Standard. Washington, DC: Earthscan. Recuperado de https://waterfootprint.org/media/downloads/ TheWaterFootprintAssessmentManual_2.pdf

Hurtado González, M. (2015). La regulación del servicio público domiciliario (SDP) de agua en Colombia. (Tesis de grado). Universidad del Valle, Santiago de Cali, Colombia. Recuperado de http://bibliotecadigital.univalle.edu.co/ handle/10893/9273

Instituto de Hidrología, Meteorología y Estudios Ambientales (IDEAM). (2015). Estudio nacional del agua 2014. Bogotá: IDEAM. Recuperado de http://documentacion. ideam.gov.co/openbiblio/bvirtual/023080/ENA_2014.pdf.

Junca, J. (2000). Determinación del consumo básico de agua potable subsidiable en Colombia. [Serie Archivos de Macroeconomía, n. ${ }^{\circ}$ 139]. Bogotá: Departamento Nacional de Planeación.. Recuperado de: https://colaboracion.dnp.gov.co/CDT/ Estudios\%20Econmicos/139.pdf

Lee, T. y Jouravlev, A. (1998). Los precios, la propiedad y los mercados en la asignación del agua. [Serie Medio Ambiente y Desarrollo, n. ${ }^{\circ} 6$ ]. Santiago de Chile: CEPAL. Recuperado de https://repositorio.cepal.org/bitstream/handle/11362/5732/ S9800051_es.pdf? sequence $=1$ \&isAllowed $=y$.

Luengo M., A. (2015). El derecho a la ciudad y su naturaleza jurídico-urbanística. Los supuestos de Barakaldo y Milton Keynes. (Tesis doctoral). Universidad Complutense de Madrid, Madrid, España. Recuperado de https://eprints.ucm.es/34446/1/ T36721.pdf.

Ministerio de Ambiente, Vivienda y Desarrollo Territorial (MAVDT). (2010). Política Nacional para la Gestión Integral del Recurso Hídrico. Bogotá: MAVDT. Recuperado de http://www.minambiente.gov.co/images/ GestionIntegraldelRecursoHidrico/pdf/Presentaci\%C3\%B3n_Pol\%C3\%ADtica_ Nacional___Gesti\%C3\%B3n_/libro_pol_nal_rec_hidrico.pdf.

Ministerio de Vivienda, Ciudad y Territorio. (2012). Reglamento Técnico del Sector de Agua Potable y Saneamiento Básico (RAS). Título D: Sistemas de recolección y evacuación de aguas residuales domésticas y aguas lluvias. (2ª ed.). Bogotá, D.C.: Viceministerio de Agua y Saneamiento Básico (ed.). Universidad de los Andes, Centro de Investigaciones en Acueductos y Alcantarillados (Ciacua) (consultor). Recuperado de http://www.minvivienda.gov.co/Documents/ViceministerioAgua/ TITULO_D.pdf.

Motta, R. (2011). El derecho al agua potable en la jurisprudencia colombiana. Revista Republicana, 11, 53-67. Recuperado de http://revista.urepublicana.edu.co/ 
wp-content/uploads/2012/07/El-Derecho-al-agua-potable-en-la-jurisprudenciacolombiana.pdf

Olivan, H. (2007). Manual básico de derecho urbanístico. España: Grau Asociados Abogados. Recuperado de http://www.grauasociados.com/textos/manual.pdf

Organización Mundial de la Salud (OMS). (2009). Cantidad mínima de agua necesaria para uso doméstico. [Guías técnicas sobre saneamiento, agua y salud. Guía técnica n. ${ }^{\circ}$ 9]. Recuperado de http://www.disaster-info.net/Agua/pdf/9-UsoDomestico.pdf

Parques Nacionales Naturales de Colombia. (2015). Registro Único Nacional de áreas protegidas (RUNAP). Recuperado de http://runap.parquesnacionales.gov.co/cifras

Pirez, P. (2000). Servicios urbanos y equidad en América Latina: un panorama con base en algunos casos. Santiago de Chile: CEPAL.

Ramírez, D. (Diciembre de 2014). Análisis de las pérdidas de agua en los sistemas de abastecimiento. Boletín de Dinámica de Sistemas. Recuperado de http://dinamicade-sistemas.com/revista/1214g.htm

Sánchez, L. D., Flórez, M. E. y Mejía, P. A. (2002). Caracterización de pérdidas de agua en el sistema de distribución del acueducto El Retiro. En Conferencia Internacional Usos Múltiples del Agua: Para la Vida y el Desarrollo Sostenible (pp. 116-123). Cali: Universidad del Valle.

Sanabria, T. (2016). Correspondencias entre consumo de suelo y la atención de necesidades. Una aproximación de la planeación territorial desde el enfoque de derechos. [Documento de trabajo]. Bogotá: Universidad de América.

Sistema de Información Ambiental (SIAC). (2002). Perfil del estado de los recursos naturales y del medio ambiente en Colombia 2001. (Tomo 3). Bogotá, D. C.: IDEAM. Recuperado de http://documentacion.ideam.gov.co/openbiblio/bvirtual/002592/ TOMO3-PERFIL.pdf

Solá-Morales, M. (1997). Las formas de crecimiento urbano. Barcelona: Ediciones UPC.

Unidad de Planeación Minero-Energética (UPME). (2013). Proyección de demanda de energía en Colombia. Bogotá: Ministerio de Minas y Energía.

World Bank Group. (2002). Ciudades en movimiento: revisión de la estrategia de transporte urbano del Banco Mundial. Recuperado de http:// documentos.bancomundial.org/curated/es/876011468142795915/ pdf/249100a1spanish1 cities 1 on 1 the 1 move.pdf 